\title{
LAS ACTITUDES RELACIONADAS CON LA CIENCIA Y LA TECNOLOGÍA EN EL ESTUDIO PISA 2006
}

\author{
José Antonio Acevedo Díaz \\ Consejería de Educación de la Junta de Andalucía. \\ Inspección de Educación. Delegación Provincial de Huelva. \\ E-mail: ja_acevedo@vodafone.es
}

[Recibido en Febrero de 2007, aceptado en Mayo de 2007]

\begin{abstract}
RESUMEN(Inglés)
Este artículo describe las principales novedades incorporadas en la evaluación comparativa internacional PISA 2006. Después de analizar el significado de alfabetización científica adoptado, se presta especial atención a la evaluación de actitudes relacionadas con la ciencia y la tecnología, a señalar los aspectos que evalúa esta nueva dimensión y a cómo lo hace el proyecto PISA 2006.
\end{abstract}

Palabras clave: Evaluación externa; proyecto PISA 2006; alfabetización científica; actitudes relacionadas con la ciencia y la tecnología.

\section{INTRODUCCIÓN}

Las evaluaciones educativas externas están bastante extendidas y consolidadas en la actualidad y se les suele conceder gran valor en la mayoría de los países del mundo. Como se ha señalado en otras ocasiones, cuando están mal planteadas son un lastre para las innovaciones curriculares (Oliva y Acevedo, 2005), pero si están bien concebidas y planificadas, pueden contribuir a orientar la enseñanza hacia aquellos enfoques e innovaciones que están más en sintonía con las aportaciones de la investigación educativa (Acevedo 2005b; Gil-Pérez y Vilches, 2006b).

Entre los diversos tipos de evaluaciones externas, hay que destacar los estudios internacionales para la comparación del rendimiento que logran los estudiantes. No son estudios de carácter académico, sino que están dirigidos sobre todo a los gestores y administradores de la educación - policy oriented studies- con el fin de ayudarles a tomar decisiones en su propio ámbito para facilitar el impulso y la orientación de las reformas de la enseñanza. A partir de las fortalezas y debilidades detectadas, estos estudios ofrecen la oportunidad de ampliar y enriquecer la visión nacional, regional o local, proporcionando un contexto más amplio en el que interpretar los resultados alcanzados. Además, suministran las pruebas empíricas que sirven de apoyo a las correspondientes deliberaciones teóricas e ideológicas. Estos estudios también aportan diversos instrumentos para el seguimiento de los niveles de rendimiento escolar por 
parte de las autoridades educativas, incluso en aquellos casos en los que las competencias educativas están descentralizadas. Por último, son útiles para detectar los aspectos fuertes y débiles del currículo y orientar a los centros educativos. Con los incentivos apropiados, los estudios comparativos internacionales pueden servir para motivar la mejora del aprendizaje de los estudiantes, la enseñanza practicada por el profesorado y la eficacia global de los centros educativos. Tales estudios tienen, pues, un indudable valor porque permiten aprender de los demás, entender mejor que la educación se basa casi siempre en preferencias políticas y tradiciones culturales, y comprender que la realidad propia puede ser diferente (Schreiner y Sjøberg, 2004b).

Junto a las luces señaladas, en las evaluaciones comparativas internacionales también hay algunas sombras, que son capaces de dificultar la comparación entre países y poner en entredicho algunas de las afirmaciones de los informes derivados de estos estudios. Así, estas evaluaciones suelen recibir frecuentes críticas por los sesgos que pueden incluir en sus marcos teóricos de referencia, en los instrumentos de evaluación (pruebas y cuestionarios) y en ciertos aspectos metodológicos, lo que condicionaría los resultados y produciría efectos indeseables. Por ejemplo, el desarrollo y la selección de las preguntas deben prestar una atención especial a las diferencias interculturales, tanto para intentar asegurar la validez de la evaluación como para respetar los diferentes intereses, valores y tradiciones de los distintos países participantes (SolanoFlores y Nelson-Barber, 2001). Sin embargo, es fácil comprobar que a veces hay cierta tendencia a privilegiar la cultura anglosajona en estas pruebas. Así mismo, también pueden surgir otros problemas debido a los sesgos que la traducción de la redacción original de las pruebas -generalmente en inglés y francés- puede introducir (Solano-Flores, Contreras-Niño y Backhoff-Escudero, 2006).

De todos los estudios comparativos internacionales puestos en marcha en los últimos años para evaluar los resultados del aprendizaje escolar en ciencias -junto a lectura y matemáticas- el más mencionado actualmente tal vez sea el proyecto PISA ${ }^{1}$ de la $\mathrm{OCDE}^{2}$, un estudio en el que España ${ }^{3}$ ha participado en las tres aplicaciones realizadas hasta ahora en los años 2000, 20034 y $2006^{5}$.

Como las características generales del estudio PISA ya han sido expuestas en un trabajo anterior (Acevedo, 2005b) ${ }^{6}$, aquí sólo se resumen las principales en la tabla 1.

\footnotetext{
${ }^{1}$ Acrónimo de su nombre en inglés (Programme for International Student Assessment).

2 Organización para la Cooperación y Desarrollo Económico (Organization for Economic Cooperation and Development, OECD en inglés).

${ }^{3}$ El Ministerio de Educación y Ciencia -antes Ministerio de Educación, Cultura y Deporte- ha llevado a cabo las tareas de coordinación e implantación de PISA en España por medio del Instituto de Evaluación -primero denominado Instituto Nacional de Calidad y Evaluación (INCE) y luego Instituto Nacional de Evaluación y Calidad del Sistema Educativo (INECSE) - y en estrecha colaboración con las Administraciones Educativas de las Comunidades Autónomas.

${ }^{4}$ Las Comunidades Autónomas de Castilla y León, Cataluña y País Vasco participaron en PISA 2003 con una muestra ampliada, lo que permitió la presentación desglosada de sus resultados (véase, p.e., el número extraordinario dedicado a PISA por la Revista de Educación, 2006; en concreto las páginas 431-474).

${ }^{5}$ Además de las tres indicadas en la nota anterior, siete Comunidades Autónomas más han ampliado su muestra en PISA 2006 para poder obtener resultados propios: Andalucía, Aragón, Asturias, Cantabria, Galicia, La Rioja y Navarra (véase, p.e., el número extraordinario dedicado a PISA por la Revista de Educación, 2006; en concreto las páginas 477-542).

6 En ese mismo artículo se citan varias referencias bibliográficas que dan cuenta de las características del proyecto PISA con mucho detalle, a las que hay que añadir las más reciente de Pajares (2005), así como el número extraordinario dedicado a PISA por la Revista de Educación (2006) mencionado en las notas 4 y 5.
} 
Un detalle más de la evaluación PISA a tener en cuenta es que también se pregunta a los estudiantes, mediante la administración de un cuestionario general, por sus motivaciones hacia el aprendizaje, sus sentimientos sobre sí mismos, sus estrategias de aprendizaje, las características de sus centros de enseñanza y sus ambientes familiares; todo ello con el objetivo de indagar en algunas de las principales características asociadas al éxito escolar. En cambio, no se administra un cuestionario equivalente al profesorado, pues la muestra no se refiere a un curso o grupo-aula en particular. Esta limitación incide muy negativamente en las posibilidades de análisis de la relación entre las prácticas didácticas o el clima del aula y los resultados de rendimiento.

\begin{tabular}{|c|c|c|}
\hline \multicolumn{3}{|c|}{ PISA es un programa de evaluación internacional: } \\
\hline$\sqrt{ }$ & Comparativo. & $\begin{array}{l}\text { La comparación internacional }{ }^{7} \text { entre diversos sistemas } \\
\text { educativos permite una aceptable medición relativa del } \\
\text { rendimiento de un sistema educativo particular en } \\
\text { determinadas áreas de conocimiento. }\end{array}$ \\
\hline$\sqrt{ }$ & $\begin{array}{l}\text { Centrado en tres áreas de } \\
\text { conocimiento básicas. }\end{array}$ & Lectura, matemáticas y ciencias. \\
\hline$\sqrt{ }$ & $\begin{array}{l}\text { Con periodicidad cíclica en el } \\
\text { tiempo. }\end{array}$ & $\begin{array}{l}\text { Cada tres años se evalúan las tres áreas, prestándose } \\
\text { especial atención a una de ellas: lectura en el año 2000, } \\
\text { matemáticas en } 2003 \text { y ciencias en } 2006^{8} \text {. }\end{array}$ \\
\hline$\sqrt{ }$ & $\begin{array}{l}\text { Orientado a competencias } \\
\text { necesarias para la vida adulta. }\end{array}$ & $\begin{array}{l}\text { El objetivo es evaluar la alfabetización ("literacy") o } \\
\text { preparación acumulada en la educación obligatoria (hasta } \\
\text { los } 15 \text { años de edad) para afrontar los retos cotidianos de } \\
\text { la vida adulta. PISA no es un proyecto rigurosamente } \\
\text { vinculado al currículo, lo que en cierto modo facilita la } \\
\text { comparación entre países con sistemas educativos que } \\
\text { tienen una organización distinta. }\end{array}$ \\
\hline$\sqrt{ }$ & $\begin{array}{l}\text { Destinado a la toma de } \\
\text { decisiones en política } \\
\text { educativa. }\end{array}$ & $\begin{array}{l}\text { Más orientado a proporcionar datos y extraer conclusiones } \\
\text { relevantes para la dirección global del sistema educativo } \\
\text { que a analizar los procesos de enseñanza y aprendizaje que } \\
\text { tienen lugar en las aulas. }\end{array}$ \\
\hline
\end{tabular}

Tabla 1.- Principales características del proyecto PISA.

De manera general, este artículo trata de la evaluación de la alfabetización científica (scientific literacy $)^{9}$ que realiza el proyecto PISA y, de modo más concreto, se centra

\footnotetext{
${ }^{7}$ En la evaluación PISA 2000 participaron 32 países (28 de la OCDE y 4 que no eran miembros), a los que se añadieron 11 más en una evaluación especial realizada el año 2002. En PISA 2003 participaron los 30 países miembros de la OCDE: Alemania, Australia, Austria, Bélgica, Canadá, Corea, Dinamarca, España, Estados Unidos, Francia, Finlandia, Grecia, Holanda, Hungría, Irlanda, Italia, Islandia, Japón, Luxemburgo, México, Noruega, Nueva Zelanda, Polonia, Portugal, Reino Unido -su índice de respuesta fue demasiado bajo para asegurar la comparación con los demás países participantes-, República Checa, República Eslovaca, Suecia, Suiza y Turquía, y otros 11 países asociados: Brasil, Rusia, Hong Kong - China, Indonesia, Letonia, Liechtenstein, Macao - China, Serbia y Montenegro -no se dispusieron datos de Montenegro-, Tailandia, Túnez y Uruguay. En el tercer estudio llevado a cabo en 2006 han participado 56 países.

${ }^{8}$ De este modo, cada nueve años se cierra un ciclo. Esta actualización de resultados permite a los países participantes hacer un seguimiento cíclico de los progresos en cada área. Aunque este período de tiempo puede parecer dilatado, hay que tener en cuenta que los cambios en educación son siempre lentos.

9 Las versiones españolas de las publicaciones de la OCDE relativas a PISA 2000 tradujeron el término "scientific literacy" como "formación científica" (OECD, 2000), mientras que las posteriores correspondientes a PISA 2003 y 2006 lo hacen como "competencia científica" (OECD, 2003a; OECD, 2006). En este artículo, se prefiere conservar la expresión "alfabetización científica", porque está ampliamente extendida en la bibliografía de didáctica de las ciencias. La alfabetización científica implica, por supuesto, formación y la posesión de distintas capacidades y competencias, tal y como se verá en este artículo.
} 
en la evaluación de las actitudes relacionadas con la ciencia y la tecnología realizada por PISA 2006, donde el área de ciencias ha sido por primera vez el principal objeto de la evaluación. Antes de entrar en el análisis de la evaluación de las actitudes, se dedicará la siguiente sección a describir el marco teórico que establece la definición de alfabetización científica adoptada por PISA.

\section{LA ALFABETIZACIÓN CIENTÍFICA EN LOS ESTUDIOS PISA}

El estudio PISA parte de la idea de alfabetización como un conjunto de competencias que se debe poseer para resolver diferentes tareas en diversos contextos de interés personal, social y mundial, así como la comprensión de conceptos básicos necesarios para conseguirlo. En consecuencia, PISA 2000 definió la alfabetización científica como:

"La capacidad de emplear el conocimiento científico para identificar cuestiones y obtener conclusiones a partir de pruebas, con el fin de comprender y ayudar a tomar decisiones acerca del mundo natural y de los cambios artificiales que produce en él la actividad humana."

(OECD, 2000, p. 115 de la traducción castellana, 2001).

Salvo por pequeñas diferencias en la traducción, la definición anterior se repitió en el estudio PISA 2003 (véase OECD, 2003a, p. 115 de la traducción castellana, 2004). Esta forma de entender la alfabetización científica se corresponde aproximadamente con el tercero de los cuatro niveles -nominal, funcional, conceptual y procedimental, y multidimensional- del modelo continuo desarrollado por Bybee (1997) en su análisis seminal de la alfabetización científica; esto es, se trata de una alfabetización científica que es básicamente conceptual y procedimental (Acevedo, 2005b; Acevedo, Vázquez y Manassero, 2003; Gutiérrez, 2006).

En los estudios PISA 2000 y 2003 la definición de la alfabetización científica se caracteriza por tres dimensiones relacionadas entre sí: (i) capacidades o destrezas científicas, (ii) conceptos o conocimientos científicos y (iii) contextos de aplicación del conocimiento científico. Estas dimensiones continúan presentes en PISA 2006.

Las capacidades o destrezas hacen referencia a los procedimientos científicos necesarios para la resolución de una cuestión. Como la mayoría de los estudiantes no serán científicos en el futuro, se da prioridad a los procedimientos relacionados con el funcionamiento de la ciencia frente a los utilizados en la ciencia. Así, se considera que las personas necesitan saber cuándo es pertinente usar el conocimiento científico y discernir entre las preguntas que la ciencia puede responder y aquellas a las que no puede dar respuesta. También tienen que ser capaces de juzgar cuándo una prueba científica es válida, tanto en lo relativo a su pertinencia como al modo en que ha sido obtenida. Por último, y quizás sea lo más importante, las personas necesitan tener la capacidad de relacionar los hechos y los datos con las conclusiones obtenidas a partir de ellos, así como sopesar las pruebas a favor y en contra de determinadas acciones que pueden afectar a su vida personal o familiar, a su comunidad local, a su país o a todo el planeta. En resumen, el enfoque adoptado por el estudio PISA no se centra tanto en cómo "hacer ciencia" ni en cómo "crear conocimientos científicos", lo cual se estima que tal vez debería reservarse a la minoría de estudiantes que serán científicos en el futuro, sino en cómo "funciona la ciencia"; esto es, en la capacidad de evaluar 
las pruebas aportadas, distinguir entre teorías y observaciones, y valorar el grado de confianza que hay que conceder a las explicaciones aportadas (Millar y Osborne, 1998).

Los conceptos científicos empleados en la evaluación PISA se corresponden con la comprensión conceptual requerida para la aplicación de los procedimientos científicos en determinados contextos. Estos conceptos proceden de la biología, la física, la química, las ciencias de la Tierra y el espacio, y la tecnología. Su selección se basa en tres criterios de relevancia: (i) utilidad en situaciones de la vida real, (ii) duración en el tiempo por su relación con la ciencia que se prevé importante en el futuro próximo, y (iii) adecuación al nivel de desarrollo del alumnado de 15 años.

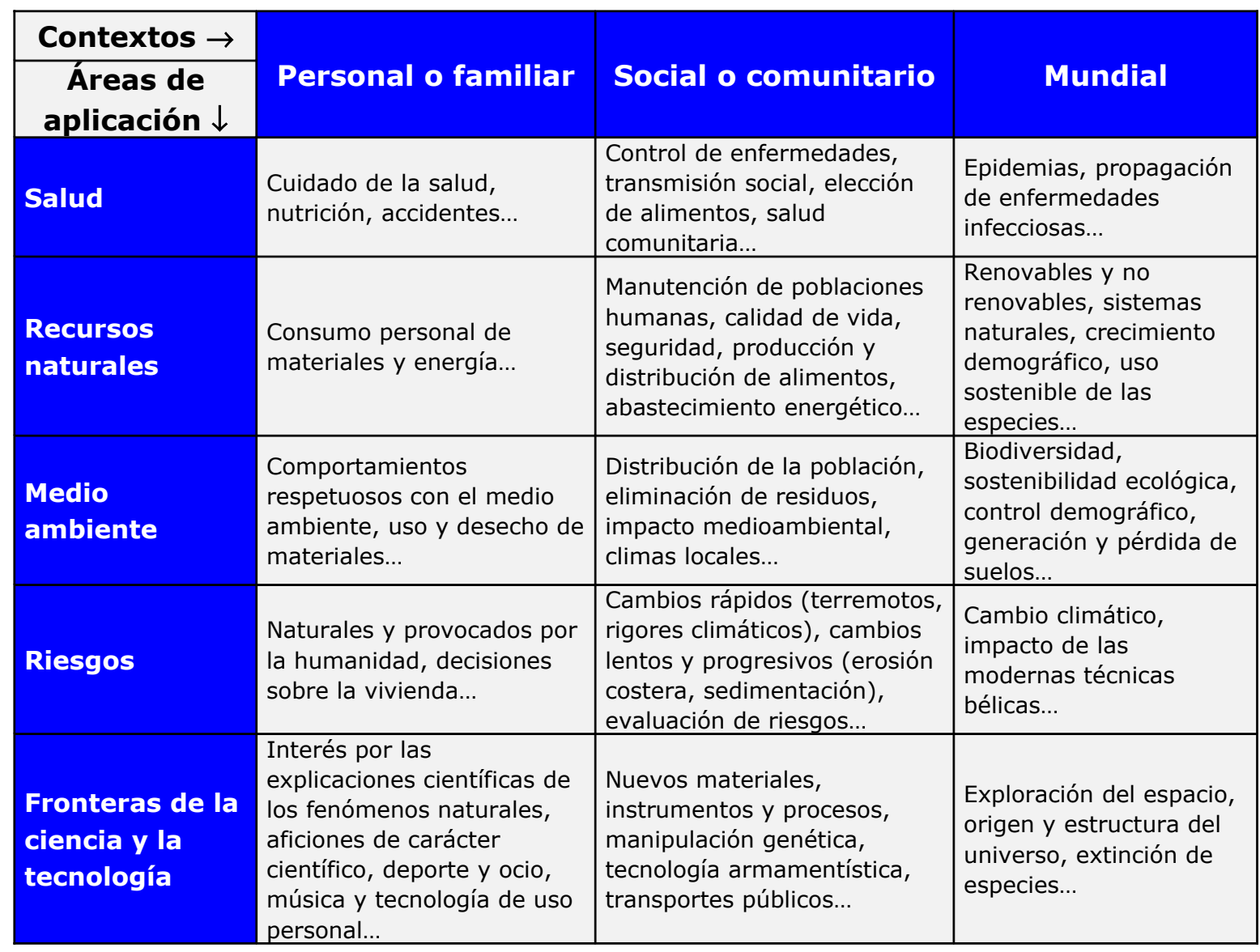

Tabla 2.- Ejemplos correspondientes a PISA de diversas aplicaciones de la ciencia y la tecnología en tres contextos relevantes (OECD, 2006).

Los contextos son los marcos concretos donde se presentan las diversas situaciones relacionadas con la ciencia y la tecnología, en las que se aplican las destrezas y los conceptos. Como contexto científico para las preguntas, PISA da preferencia al más cotidiano de la vida real antes que a los más académicos del aula o las prácticas de laboratorio. Las situaciones del mundo real usadas en las cuestiones de ciencias de los estudios PISA se refieren a asuntos relevantes desde un punto de vista (i) personal o familiar (p.e., cuestiones de alimentación, salud, consumo...), (ii) social o comunitario (p.e., instalación de una central eléctrica o un vertedero industrial, abastecimiento y depuración de aguas, transporte público...) y (iii) mundial (p.e., calentamiento global 
del planeta, disminución de la biodiversidad, crecimiento demográfico...). En algunas ocasiones también se recurre a un contexto histórico (p.e., evolución del conocimiento científico, influencia de la sociedad en la ciencia y, viceversa, de la ciencia en la sociedad...), tecnológico, etc. En la tabla 2 puede verse un listado de ejemplos de situaciones correspondientes a diversas áreas de aplicación y a los tres primeros tipos de contextos mencionados, que son los más frecuentes.

La definición de alfabetización científica de PISA 2006 concuerda conceptualmente con la establecida en las dos anteriores aplicaciones de PISA (OECD, 2000, 2003a); no obstante, se han introducido algunos cambios sustanciales en esta ocasión. Por un lado, la definición se ha reelaborado haciendo más hincapié en la comprensión de la naturaleza de la ciencia y la metodología científica -conocimiento sobre la ciencia-, así como en el papel de la tecnología en la ciencia contemporánea y las relaciones entre ambas. Por otro lado, la definición también se ha ampliado con la inclusión explícita de diferentes aspectos relativos a la actitud del alumnado ante determinadas cuestiones con relevancia científica y tecnológica (Fensham 2004).

Los cambios conceptuales se han realizado para resaltar algunos aspectos que estaban implícitos en la definición de PISA 2000 y 2003, como son el conocimiento sobre la ciencia (Acevedo et al., 2007; Bartholomew, Osborne y Ratcliffe, 2004; Bell, 2005; Driver et al., 1996; Lederman, 2006; McComas, 1998; Millar y Osborne, 1998; Osborne et al., 2003; Vázquez et al., 2001; entre otros) y las relaciones mutuas entre la ciencia y la tecnología (Acevedo 1996, 2006b; Acevedo y Vázquez, 2003; Acevedo et al., 2003; Cajas, 2001; Gardner, 1990, 1994, 1999; Gilbert, 1992; Maiztegui et al., 2002; Valdés et al., 2002; entre otros). Mientras que en PISA 2000 y 2003 los conocimientos de ciencia y sobre la ciencia no estaban diferenciados en la noción de conocimiento científico, PISA 2006 desglosa el segundo aspecto y lo desarrolla con la inclusión de dos términos -la investigación científica y las explicaciones científicaspara poner de relieve la comprensión que el alumnado tiene de los principales rasgos de la ciencia, entendida ésta como una forma de conocimiento e investigación, lo que es un importante elemento de la alfabetización científica. Por otra parte, puesto que la ciencia y la tecnología están estrechamente relacionadas en el mundo contemporáneo, también se ha mejorado la definición en PISA 2006 añadiendo el conocimiento de las relaciones entre la ciencia y latecnología, otro aspecto relevante de la alfabetización científica que, si bien no estaba desarrollado, se presuponía implícito con anterioridad (OECD, 2006).

En suma, la alfabetización científica está bastante más elaborada en PISA 2006 que en las veces anteriores, donde no fue el área principal de evaluación. En esta ocasión se ha definido como:

"Los conocimientos científicos de un individuo y el uso de ese conocimiento para identificar problemas, adquirir nuevos conocimientos, explicar fenómenos científicos y extraer conclusiones basadas en pruebas sobre cuestiones relacionadas con la ciencia. Asimismo, comporta la comprensión de los rasgos característicos de la ciencia, entendida como un método del conocimiento y la investigación humanas, la percepción del modo en que la ciencia y la tecnología conforman nuestro entorno material, intelectual y cultural, y la disposición a implicarse en asuntos relacionados con la ciencia y con las ideas de la ciencia como un ciudadano reflexivo."

(OECD, 2006, p. 12; p. 13 de la traducción castellana, 2006). 


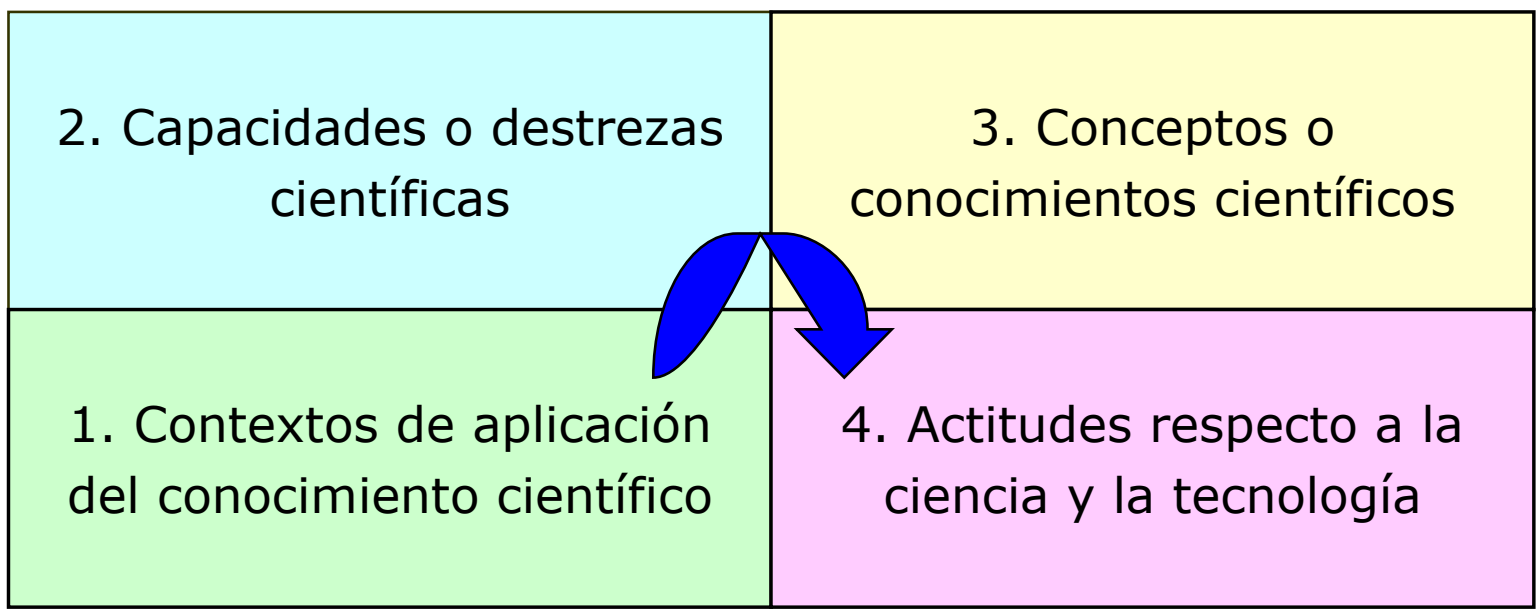

Figura 1.- Dimensiones correspondientes a la evaluación de la alfabetización científica PISA 2006.

La alfabetización científica correspondiente a PISA 2006 hace referencia, pues, a las cuatro siguientes dimensiones relacionadas entre sí (véase la figura 1): (i) contextos de las cuestiones, (ii) capacidades o destrezas, (iii) conceptos o conocimientos científicos y (iv) actitudes relacionadas con la ciencia. Como se ha indicado más arriba, las tres primeras ya estaban presentes en PISA 2000 y 2003, aunque ahora se han reelaborado, mientras que la cuarta es nueva (véase la tabla $3^{10}$ ).

\begin{tabular}{|c|c|c|c|}
\hline Contextos & Capacidades & Conceptos & Actitudes \\
\hline $\begin{array}{l}\text { Situaciones de la vida que } \\
\text { involucran a la ciencia y la } \\
\text { tecnología en cinco áreas } \\
\text { de aplicación: } \\
\text { 1. Salud. } \\
\text { 2. Recursos materiales. } \\
\text { 3. Medio ambiente. } \\
\text { 4. Riesgos. } \\
\text { 5. Fronteras de la ciencia y } \\
\text { la tecnología. }\end{array}$ & $\begin{array}{l}\text { 1. Identificar cuestiones } \\
\text { científicas. } \\
\text { 2. Explicar fenómenos } \\
\text { aplicando los } \\
\text { conocimientos científicos. } \\
\text { 3. Utilizar pruebas } \\
\text { científicas para tomar y } \\
\text { comunicar decisiones bien } \\
\text { documentadas. }\end{array}$ & $\begin{array}{l}\text { 1. Conocimientos } \\
\text { científicos del mundo } \\
\text { relativos a sistemas (i) } \\
\text { físicos, (ii) vivos, (iii) de la } \\
\text { Tierra y el espacio, y (iv) } \\
\text { tecnológicos. } \\
\text { 2. Conocimientos sobre la } \\
\text { ciencia (naturaleza de la } \\
\text { ciencia) acerca de (i) la } \\
\text { investigación científica (los } \\
\text { medios) y (ii) las } \\
\text { explicaciones científicas } \\
\text { (los objetivos). }\end{array}$ & $\begin{array}{l}\text { 1. Interés por la ciencia*. } \\
\text { 2. Apoyo a la investigación } \\
\text { científica. } \\
\text { 3. Responsabilidad ante los } \\
\text { recursos y el medio } \\
\text { ambiente }^{* \star} \text {. }\end{array}$ \\
\hline
\end{tabular}

(*) El interés por aprender acerca de la ciencia, que es uno de los aspectos del interés por la ciencia, se evalúa mediante preguntas insertadas en las cuestiones.

$(* *)$ Sólo se evalúa en el cuestionario general administrado al alumnado.

Tabla 3.- Descriptores correspondientes a las dimensiones de la evaluación de la alfabetización científica PISA 2006.

Si se analiza con el modelo de Bybee (1997), la alfabetización científica de PISA 2006 continúa siendo conceptual y procedimental sobre todo, aunque las novedades indicadas respecto a PISA 2000 y 2003 apuntan hacia una alfabetización científica en parte multidimensional, aunque aún sea manera débil (véase la figura 2 ).

\footnotetext{
${ }^{10}$ Esta idea de la alfabetización científica se asemeja al concepto utilizado en la definición y selección de competencias DeSeCo (OECD, 2003b), puesto que en ambos casos se incluyen las actitudes y los valores, además de los conceptos o conocimientos y las capacidades, destrezas o procedimientos.
} 
La innovación más significativa de la evaluación de la alfabetización científica PISA 2006 ha sido, sin duda, la inclusión de cuestiones relativas a las actitudes relacionadas con la ciencia y la tecnología, no sólo en el cuestionario general administrado a los estudiantes sino en una serie de preguntas sobre cuestiones científicas anexas a las preguntas de la prueba correspondientes a esas mismas cuestiones (Fensham, 2004; OECD, 2006). Debido a las repercusiones que se esperan de esta importante novedad, la siguiente sección se dedica específicamente a ella.

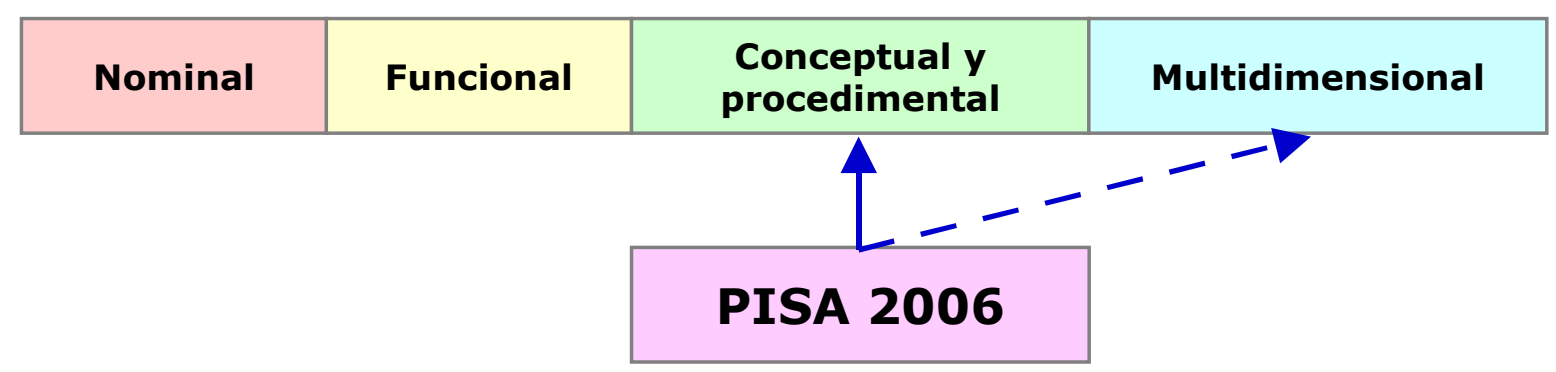

Figura 2.- Tipo de alfabetización científica que sigue PISA 2006 respecto al modelo continuo de Bybee (1997).

\section{LA ATENCIÓN DE PISA 2006 AL COMPONENTE ACTITUDINAL}

La crisis de la enseñanza de las ciencias alcanza hoy a la mayoría de los países desarrollados, especialmente en la educación secundaria (Fensham, 2002a,b; Fourez, 1999, 2002; Jenkins y Nelson, 2005). Aunque esta crisis se debe en buena parte a problemas generales de la educación, también tiene sus propios rasgos específicos, consecuencia de una finalidad propedéutica enfocada principalmente hacia los hechos y conceptos científicos (Banet, 2007), que tiende a descuidar los aspectos emotivos y afectivos propios del ámbito actitudinal (Acevedo, 2004; Aikenhead 2003a; Furió et al., 2001; Vázquez y Manassero, 2006, 2007). Un efecto muy indeseable de esta crisis de la educación científica es el continuo descenso de estudiantes en los estudios universitarios de ciencia y en las profesiones relacionadas con la ciencia, tanto por la baja elección inicial como por el frecuente abandono de los estudios debido a la enseñanza que se practica, sobre todo en la universidad pero no sólo en esa etapa (Gago, 2004). Las actitudes negativas hacia la ciencia y la tecnología, adquiridas a lo largo de toda la escolaridad, están en el origen de estas decisiones y, tal vez, deban considerarse el centro del problema (Vázquez, Acevedo y Manassero, 2005).

Las actitudes de las personas desempeñan un papel esencial a la hora de determinar su interés, atención y reacciones hacia la ciencia y la tecnología en general, y hacia temas concretos relacionados con ellas en particular (Fensham, 2004). Un objetivo fundamental de la educación científica es que los estudiantes desarrollen una serie de actitudes que promuevan su interés por los temas científicos, así como la adquisición y aplicación del conocimiento científico y tecnológico para beneficio personal y social. No obstante, como resultado del olvido de los sentimientos y las emociones por la enseñanza propedéutica de las ciencias, muchos estudiantes están insatisfechos con lo que hacen en las aulas de ciencia, particularmente en los sistemas educativos de los 
países más desarrollados del ámbito cultural occidental. En estos países, la ciencia escolar suele considerarse difícil, aburrida, impersonal, desconectada de los intereses de los estudiantes e irrelevante para la sociedad en su conjunto, lo cual origina que la mayoría del alumnado rechace los cursos de ciencias y que gran parte del desencanto hacia la ciencia y la tecnología tenga su raíz en la propia escuela (Fensham, 2002a,b, 2005; Osborne, Simon y Collins, 2003; Reiss, 2006; Vázquez, Acevedo y Manassero, 2005; Vázquez y Manassero, 2006, 2007) ${ }^{11}$.

Como respuesta a ello, y novedad de gran relieve, el diseño de PISA 2006 dedica una atención especial a la evaluación de diversas actitudes relacionadas con la ciencia, la tecnología y el medio ambiente, dando así cabida a los aspectos afectivos y emotivos (Fensham, 2004; OECD, 2006), que constituyen un componente esencial de la alfabetización científica porque contribuyen a despertar el interés de los estudiantes y a mantener su apoyo a la ciencia, a la vez que los motivan a actuar (Schibeci, 1984). En efecto, la alfabetización científica de una persona también implica una serie de actitudes, creencias, motivaciones, valores y, en última instancia, comportamientos y acciones (Acevedo, 2006c; Acevedo et al., 2005), una posición que sostiene con vigor el movimiento CTS en el aprendizaje de las ciencias (Acevedo, Vázquez y Manassero, 2002, 2003; Aikenhead, 2003b, 2005a,b).

La inclusión de las actitudes y de las áreas seleccionadas para su evaluación en PISA 2006 está basada en la taxonomía del dominio afectivo en la educación científica realizada hace tres décadas por Klopfer (1976), la cual es consecuencia de un trabajo anterior que supuso un temprano y notable esfuerzo del autor para intentar aclarar el carácter multidimensional característico del dominio afectivo (Klopfer, 1971). En efecto, Klopfer (1971) clasificó en ese artículo diversos aspectos afectivos relacionados con la educación científica del modo que se muestra en la tabla 4.

Un estudio que también ha influido mucho en la concepción de las actitudes respecto a las ciencias que aborda PISA 2006 es el de Gardner (1975), donde se hace una distinción fundamental y básica del constructo relativo a la actitud en dos categorías: (i) actitudes hacia la ciencia y (ii) actitudes científicas.

Las actitudes hacia la ciencia vendrían a ser los sentimientos, creencias y valores que se sostienen acerca de diversos objetos de actitud, tales como la empresa científica, la ciencia escolar, los propios científicos, el impacto de la ciencia en la sociedad, etc. En el caso de la ciencia escolar, estas actitudes incluirían en particular las disposiciones, tendencias o inclinaciones a responder a los diversos elementos implicados en el aprendizaje de la ciencia. Gardner (1975) señaló tres componentes de estas actitudes: (i) el interés por los contenidos científicos, (ii) las actitudes hacia los científicos y su trabajo, y (iii) las actitudes hacia los resultados obtenidos por la ciencia. Como han apuntado Vázquez y Manassero (1995), las actitudes hacia la ciencia ponen el acento especialmente en el componente emotivo de las actitudes, mientras que las actitudes

\footnotetext{
${ }^{11}$ El proyecto comparativo internacional ROSE ("The Relevance of Science Education"), dirigido por Svein Sjøberg de la Universidad de Oslo, tiene como principal finalidad el estudio de la relevancia de la educación científica para estudiantes de secundaria pertenecientes a diferentes contextos culturales (Acevedo, 2005a; Jenkins, 2006b; Schreiner y Sjøberg, 2004b; Sjøberg, 2004; Sjøberg y Schreiner, 2005a,b,c). LoS resultados conseguidos por el proyecto ROSE en distintos países han dado lugar a diversas publicaciones acerca del interés del alumnado por la ciencia en general (Jenkins, 2006a; Schreiner, 2006; Vázquez y Manassero, 2004), la biología (Trumper, 2006a), la física (Lavonen et al., 2005; Trumper, 2006b) y la química (Vázquez y Manassero, 2006).
} 
científicas se centran más en el componente cognitivo (Osborne, Simon y Collins, 2003). Estas últimas vienen dadas por el conjunto de rasgos que las diversas metodologías de la ciencia imponen idealmente a las actividades de investigación científica -curiosidad, apertura de mente, creatividad, escepticismo, imparcialidad, honradez, objetividad, racionalidad, etc.-. En una revisión seminal de las actitudes científicas, Gauld y Hukins (1980) propusieron posteriormente una clasificación de las actitudes científicas en tres grupos principales: (i) actitud general ante las ideas y la información científicas, (ii) actitudes relativas a la evaluación de las ideas y la información científica, y (iii) compromiso con determinadas creencias científicas. Sin embargo, es necesario resaltar que la diferenciación de las actitudes científicas en elementos independientes es muy complicada debido, por una parte, al solapamiento entre los propios elementos $\mathrm{y}$, por otra, al carácter provisional y cambiante del significado epistemológico de la propia metodología científica.

Manifestar actitudes favorables hacia la ciencia y los científicos.

Aceptar la investigación científica como una forma de pensamiento.

Adoptar "actitudes científicas".

Disfrutar aprendiendo ciencia.

Interesarse por la ciencia y las actividades relacionadas con la ciencia.

Interesarse por hacer una carrera científica o ejercer un trabajo relacionado con la ciencia.

Tabla 4.- Aspectos afectivos de la educación científica según Klopfer (1971).

Otros trabajos relativos a la investigación de las actitudes relacionadas con la ciencia que han servido de referencia al marco teórico de PISA 2006 son las revisiones hechas por Haladyna y Shaughnessy (1982), Laforgia (1988) y Schibeci $(1984)^{12}$, en las que se asume la distinción de Gardner (1975) entre actitudes hacia la ciencia y actitudes científicas.

De las diversas dimensiones de las actitudes relacionadas con la ciencia, la evaluación PISA 2006 de la alfabetización científica se ocupa de tres: (i) interés por la ciencia, (ii) apoyo a la investigación científica y (iii) responsabilidad respecto a los recursos y el medio ambiente. Mediante la valoración de estas dimensiones se espera disponer de una extensa base de datos internacional acerca del interés general de los estudiantes por la ciencia, sus actitudes y valores específicamente científicos, y su responsabilidad respecto a una serie de cuestiones relativas a la ciencia y la tecnología con amplias implicaciones nacionales y mundiales. Sin embargo, hay que advertir que no se han evaluado las actitudes del alumnado respecto a los profesores o a los currículos escolares de ciencias. Así mismo, se pretende que los resultados que se consigan sirvan para obtener suficiente información respecto al problema que supone el creciente descenso del interés por diversos estudios de ciencias entre la juventud.

El interés por la ciencia se ha elegido por su posible relación con el rendimiento en ciencias, con la elección de estudios y opciones profesionales relativas a la ciencia y la

\footnotetext{
12 Véanse también las revisiones posteriores realizadas por Osborne, Simon y Collins (2003) y por Vázquez y Manassero (1995). Esta última incluye una clasificación taxonómica de las actitudes relacionadas con la ciencia y la tecnología que es muy útil para la estructuración de este complejo constructo multidimensional.
} 
tecnología, y con el aprendizaje de las ciencias durante toda la vida. La relación entre el interés por la ciencia y el rendimiento en ciencias viene siendo investigada desde hace más de cuatro décadas, sin que aún se haya conseguido claramente un consenso sobre si existe o no un vínculo causal, o al menos una correlación positiva, entre ambas variables (Acevedo, 2006c; Osborne, Simon y Collins, 2003). De acuerdo con esta línea de investigación, PISA 2006 ha incluido esta dimensión actitudinal para disponer de información sobre la implicación del alumnado en temas sociales relacionados con la ciencia y la tecnología, su disposición a adquirir conocimientos y destrezas científicas y su inclinación hacia las opciones profesionales científicas y tecnológicas.

El apoyo a la investigación científica se considera uno de los objetivos fundamentales de la educación científica y, por tanto, es objeto de evaluación por PISA 2006. Se trata de un constructo similar a la "adopción de actitudes científicas" señalado por Klopfer (1971) (véase en la tabla 4). El aprecio y apoyo a la investigación científica implican que los estudiantes valoren positivamente los diversos métodos para la obtención de pruebas científicas, el pensamiento creativo y racional, la actitud crítica y la comunicación de conclusiones al enfrentarse a situaciones de la vida relacionadas con la ciencia y la tecnología. Entre los diferentes aspectos de esta dimensión incluidos en PISA 2006 están la utilización de pruebas científicas para la toma de decisiones y la valoración del papel de la lógica y la racionalidad para establecer conclusiones.

La responsabilidad respecto a los recursos disponibles y el medio ambiente es una preocupación mundial que, al mismo tiempo, tiene gran importancia económica. Las actitudes respecto a esta dimensión han sido objeto de numerosas investigaciones desde la década de los setenta del siglo XX y continúan siéndolo en la actualidad (Schreiner y Sjøberg, 2004a, 2005; Vázquez y Manassero, 2005). En diciembre de 2002, las Naciones Unidas aprobaron la resolución 57/254 que instituyó para un período de diez años, desde el 1 de enero de 2005, la Década de la Educación para el Desarrollo Sostenible de las Naciones Unidas (UNESCO, 2003) ${ }^{13}$. En el correspondiente Programa de Aplicación Internacional (International Implementation Scheme) para la mencionada década (UNESCO, 2005) ${ }^{14}$ se menciona el medio ambiente como una de las tres esferas de sostenibilidad, junto con la sociedad -que también incluye la cultura- y la economía, las cuales deberían figurar en los programas educativos relativos al desarrollo sostenible (Domèmech et al., 2006; Vilches y Gil-Pérez, 2003), más allá de ciertas interpretaciones tendenciosas (Gil-Pérez y Vilches, 2006a).

\footnotetext{
${ }^{13}$ Como señala la UNESCO: "La Década de las Naciones Unidas para la educación con miras al desarrollo sostenible pretende promover la educación como fundamento de una sociedad más viable para la humanidad e integrar el desarrollo sostenible en el sistema de enseñanza escolar a todos los niveles. La Década intensificará igualmente la cooperación internacional en favor de la elaboración y de la puesta en común de prácticas, políticas y programas innovadores de educación para el desarrollo sostenible".

${ }^{14}$ Véase al respecto la web de educadores por la sostenibilidad disponible en la OEI (Organización de Estados Iberoamericanos), http://www.oei.es/decada. También puede consultarse en Revista Eureka la sección permanente "Educación científica y sostenibilidad", iniciada a partir de su tercer volumen (Oliva, 2006, 2007) y destinada a impulsar la iniciativa para la educación por un futuro sostenible correspondiente a la década 2005-2014 (Gil-Pérez et al., 2006; Gil-Pérez, Vilches y Oliva, 2005).
} 


\section{¿Cómo estás de interesado en la siguiente información?} Marca con una $X$ una sola casilla en cada fila.

\begin{tabular}{|l|c|c|c|c|}
\hline \multicolumn{1}{|c|}{ Frases } & $\begin{array}{c}\text { Muy } \\
\text { interesado }\end{array}$ & $\begin{array}{c}\text { Algo } \\
\text { interesado }\end{array}$ & $\begin{array}{c}\text { Poco } \\
\text { interesado }\end{array}$ & $\begin{array}{c}\text { Nada } \\
\text { interesado }\end{array}$ \\
\hline $\begin{array}{l}\text { a) Saber más sobre el uso del ADN en la } \\
\text { resolución de delitos. }\end{array}$ & 1 & 2 & 3 & 4 \\
\hline $\begin{array}{l}\text { b) Aprender más acerca de cómo se hace } \\
\text { un perfil de ADN. }\end{array}$ & 1 & 2 & 3 & 4 \\
\hline $\begin{array}{l}\text { c) Comprender mejor cómo se pueden } \\
\text { resolver delitos con la ayuda de la ciencia. }\end{array}$ & 1 & 2 & 3 & 4 \\
\hline
\end{tabular}

Tabla 5.- Primer ejemplo de una cuestión liberada de PISA 2006 para evaluar la actitud del alumnado hacia la ciencia.

La evaluación PISA 2006 de la alfabetización científica adopta un enfoque innovador para medir las dimensiones de las actitudes citadas (Fensham, 2004; OECD, 2006). En efecto, no sólo se pregunta a los estudiantes lo que opinan sobre la ciencia en el cuestionario general administrado al alumnado, sino que también se incluyen en los cuadernillos que contienen las cuestiones contextualizadas utilizadas en la prueba de evaluación una serie de preguntas relativas a sus actitudes hacia los asuntos que se tratan en esas cuestiones (véase la última columna de la tabla 3). En este segundo caso, la evaluación de las actitudes se hace mediante una encuesta de opinión, empleando una escala del tipo Likert (véanse las tablas 5 y 6 ).

\begin{tabular}{|l|c|c|c|c|}
\hline \multicolumn{7}{|c|}{ ¿Cómo estás de interesado en la siguiente información? } \\
Marca con una X una sola casilla en cada fila. \\
\hline \multicolumn{1}{|c|}{ Frases } & $\begin{array}{c}\text { Muy } \\
\text { interesado }\end{array}$ & $\begin{array}{c}\text { Algo } \\
\text { interesado }\end{array}$ & $\begin{array}{c}\text { Poco } \\
\text { interesado }\end{array}$ & $\begin{array}{c}\text { Nada } \\
\text { interesado }\end{array}$ \\
\hline $\begin{array}{l}\text { a) Observar por un microscopio cómo son } \\
\text { las bacterias que provocan la caries. }\end{array}$ & 1 & 2 & 3 & 4 \\
\hline $\begin{array}{l}\text { b) Obtener información sobre el desarrollo } \\
\text { de una vacuna que prevenga la caries. }\end{array}$ & 1 & 2 & 3 & 4 \\
\hline $\begin{array}{l}\text { c) Comprender por qué los alimentos sin } \\
\text { azúcar pueden provocar caries. }\end{array}$ & 1 & 2 & 3 & 4 \\
\hline
\end{tabular}

Tabla 6.- Segundo ejemplo de una cuestión liberada de PISA 2006 para evaluar la actitud del alumnado hacia la ciencia.

Por una parte, el cuestionario general administrado al alumnado se utiliza para obtener diversos datos sobre sus actitudes en las tres dimensiones señaladas (interés por la ciencia, apoyo a la investigación científica y responsabilidad respecto a los recursos y el medio ambiente). Así mismo, permite recopilar un conjunto de datos adicionales sobre el grado de compromiso de los estudiantes con la ciencia (p.e., criterios de eficacia personal, disfrute y frecuencia con que se realizan actividades extraescolares relacionadas con la ciencia, etc.) y sobre sus puntos de vista acerca del valor de la ciencia en sus vidas (p.e., educación superior y opciones profesionales) y en la sociedad (p.e., beneficios sociales y económicos). 
Por otra parte, la presencia en las cuestiones contextualizadas de preguntas centradas en diversos aspectos personales, sociales y mundiales está destinada a conseguir información de los estudiantes respecto a su interés por adquirir conocimientos sobre la ciencia, que es uno de los aspectos básicos del interés por la ciencia, y el apoyo a la investigación científica. La inclusión de este tipo de preguntas aporta, además, un valor añadido a la evaluación porque permite obtener datos para determinar si la actitud de los estudiantes varía al ser evaluada dentro o fuera de un determinado contexto, si varía entre los diversos contextos y si existe correlación entre la actitud y el rendimiento en una unidad completa ${ }^{15}$.

\section{CONCLUSIONES}

PISA ha evaluado la alfabetización científica como área prioritaria por primera vez en el año $2006^{16}$. Como consecuencia de ello, se ha realizado una reelaboración respecto al planteamiento de PISA 2000 y 2003, con una interpretación más amplia de la materia objeto de evaluación. Por un lado, se ha hecho una descripción más detallada de la alfabetización científica. Por otro, se ha incluido una serie de preguntas de actitud anexas a la evaluación de conocimientos y destrezas. Esto último supone una novedad de gran relieve en el enfoque adoptado en la evaluación, que quizás tenga repercusión en los próximos estudios PISA. El principal propósito de esta innovación ha sido llevar a cabo una investigación para conocer el interés de los estudiantes por los asuntos que se tratan en las cuestiones planteadas en las unidades de evaluación.

La definición de alfabetización científica de PISA 2006 parte de la consideración sobre lo que deben conocer, valorar y ser capaces de hacer los estudiantes de 15 años para enfrentarse a situaciones de la vida real en el mundo actual y futuro. Un aspecto central de la definición y evaluación de la alfabetización científica son determinadas capacidades propias de la ciencia y de la investigación científica. Las competencias de los estudiantes para poner en práctica estas capacidades dependen, por una parte, de sus conocimientos científicos, tanto relativos al mundo natural como sobre la propia ciencia, y, por otra, de las actitudes que muestran hacia los temas científicos.

PISA 2006 ha evaluado, pues, los aspectos cognitivos y afectivos de la alfabetización científica de los estudiantes. Los aspectos cognitivos se refieren tanto a los conocimientos a los que han de recurrir los estudiantes como a su capacidad para usarlos del modo más adecuado posible en determinados procesos cognitivos propios de la ciencia y de las investigaciones científicas. En la evaluación se ha prestado particular atención a aquellos temas a los que el conocimiento científico puede

\footnotetext{
${ }^{15}$ Una unidad es un conjunto de preguntas relativas a un mismo contexto. Las unidades empiezan siempre con un estímulo -un texto, una tabla, un gráfico o una figura-, que establece el contexto de las diversas cuestiones que la integran. Más de la mitad de las unidades empleadas en PISA 2006 llevan incorporadas cuestiones relativas a la actitud. Los responsables de PISA permiten la difusión de algunas unidades para que sea posible apreciar el tipo de cuestiones a las que se deben enfrentar los estudiantes. Las demás se reservan para volver a administrarlas en otras ocasiones con el fin de poder equiparar las puntuaciones y permitir medir la evolución en el tiempo del rendimiento del alumnado. Pueden consultarse diferentes ejemplos de las unidades de ciencias liberadas correspondientes a PISA 2000 y 2003 en INECSE-MEC $(2005 a, b)$ y $\operatorname{OECD}(2000,2003 a)$, así como la reseña de estas unidades que se publicó en la revista Eureka (Acevedo 2006a). Los ejemplos de unidades de ciencias de PISA 2006, varios de ellos con preguntas sobre actitudes, están disponibles en OECD (2006).

${ }^{16}$ Está previsto que sus resultados sean dados a conocer el 4 de diciembre de 2007.
} 
contribuir permitiendo la implicación del alumnado en los procesos de toma de decisiones en situaciones con relevancia personal, social o mundial.

Como ha podido comprobarse en el desarrollo de este artículo, el marco teórico correspondiente al área científica de la evaluación PISA 2006 se nutre en gran medida de muchas de las principales y más recientes aportaciones de la didáctica de las ciencias, tales como: (i) orientación hacia una alfabetización científica basada en competencias frente a una enseñanza propedéutica que se ocupa sobre todo de los hechos y conceptos científicos, (ii) importancia concedida a la educación de actitudes relacionadas con la ciencia y la tecnología, (iii) inclusión explícita de contenidos correspondientes al conocimiento sobre la naturaleza de la ciencia y a las relaciones mutuas entre la ciencia y la tecnología, (iv) preferencia por los contextos relevantes de la vida real frente a los de carácter más académico, ( $v$ ) selección de conceptos científicos en función de su utilidad para afrontar situaciones de la vida actual y futura, y (vi) potenciación de una educación para la participación en la sociedad basada en la adopción y comunicación de decisiones razonadas y suficientemente documentadas respecto a asuntos científicos y tecnológicos con interés personal, cultural, profesional y social. En consecuencia, y sin temor a demasiadas dudas, puede afirmarse que, a pesar de las críticas que pudieran hacérsele (véase, p.e., Gutiérrez, 2006), PISA 2006 pertenece a ese tipo de evaluaciones externas que resultan muy útiles para promover reformas, enfoques e innovaciones de la enseñanza de las ciencias que estén en sintonía con muchos de los resultados conseguidos por la investigación internacional acerca de la educación científica (Acevedo, 2005b).

Los resultados de la evaluación PISA 2006 ofrecerán una valiosa información a los responsables de la política educativa de los diversos países participantes. Los datos obtenidos mediante el cuestionario general administrado al alumnado y las preguntas de actitud insertadas en los cuadernillos de la prueba proporcionarán información sobre la disposición de los estudiantes a adoptar comportamientos científicos competentes. Al mismo tiempo, puesto que la bibliografía presenta ciertos resultados contradictorios respecto a la correlación entre el rendimiento en ciencias y las actitudes, tal y como ya se ha mencionado en este artículo, los resultados que se obtengan también deberían servir para establecer si existe o no una correlación positiva entre el rendimiento de los estudiantes y los datos relativos a las actitudes evaluadas. Otros resultados procedentes del cuestionario general administrado al alumnado, tales como el grado de compromiso de los estudiantes con la ciencia y sus comportamientos respecto a la ciencia, también serán presentados y relacionados con el rendimiento del alumnado.

Las conclusiones que se deriven del estudio PISA 2006 podrán contribuir, sin duda, al debate sobre la calidad de la educación en general, y de la educación científica en particular. No obstante, como medida precautoria, tales conclusiones no deberían ser sacralizadas y sí en cambio interpretarse con la debida cautela. Tampoco se debería olvidar que los cambios en los sistemas educativos no se producen como simples respuestas a las reformas educativas que se legislan, sino que dependen de las políticas educativas concretas que las plasman en la realidad; además, estos cambios suelen darse con más lentitud de lo deseable. Por último, pero no menos importante, será imprescindible distinguir entre las conclusiones que razonablemente se extraigan 
de los datos suministrados por PISA 2006 y las interpretaciones que puedan hacerse de ellos, en particular de las correspondientes a relaciones de causa-efecto. Estas interpretaciones y atribuciones de causalidad suelen tener una gran carga ideológica y una importante intencionalidad política, lo que aumenta el riesgo de oscurecer el necesario debate y manipular las posteriores decisiones educativas que se tomen.

\section{REFERENCIAS BIBLIOGRÁFICAS}

ACEVEDO, J. A. (1996). La tecnología en las relaciones CTS. Una aproximación al tema. Enseñanza de las Ciencias, 14(1), 35-44.

ACEVEDO, J. A. (2004). Reflexiones sobre las finalidades de la enseñanza de las ciencias: Educación científica para la ciudadanía. Revista Eureka sobre Enseñanza y Divulgación de las Ciencias, 1(1), 3-16, http://www.apaceureka.org/revista/Larevista.htm.

ACEVEDO, J. A. (2005a). Proyecto ROSE: relevancia de la educación científica. Revista Eureka sobre Enseñanza y Divulgación de las Ciencias, 2(3), 440-447, http://www.apac-eureka.org/revista/Larevista.htm.

ACEVEDO, J. A. (2005b). TIMSS Y PISA. Dos proyectos internacionales de evaluación del aprendizaje escolar en ciencias. Revista Eureka sobre Enseñanza y Divulgación de las Ciencias, 2(3), 282-301, http://www.apaceureka.org/revista/Larevista.htm.

ACEVEDO, J. A. (2006a). Ejemplos de preguntas de ciencias del proyecto internacional PISA de la OECD. Revista Eureka sobre Enseñanza y Divulgación de las Ciencias, 3(1), 163-166, http://www.apac-eureka.org/revista/Larevista.htm.

ACEVEDO, J. A. (2006b). Modelos de relaciones entre ciencia y tecnología: un análisis social e histórico. Revista Eureka sobre Enseñanza y Divulgación de las Ciencias, 3(2), 198-219, http://www.apac-eureka.org/revista/Larevista.htm.

ACEVEDO, J. A. (2006c). Relevancia de los factores no-epistémicos en la percepción pública de los asuntos tecnocientíficos. Revista Eureka sobre Enseñanza y Divulgación de las Ciencias, 3(3), 369-390, http://www.apaceureka.org/revista/Larevista.htm.

ACEVEDO, J. A. y VÁZQUEZ, A. (2003). Editorial del monográfico "Las relaciones entre ciencia y tecnología en la enseñanza de las ciencias". Revista Electrónica de Enseñanza de las Ciencias, 2(3), editorial, http://www.saum.uvigo.es/reec/.

ACEVEDO, J. A., VÁZQUeZ, A. y MANASSERO, M. A. (2002). El movimiento Ciencia, Tecnología y Sociedad y la enseñanza de las ciencias. En Sala de Lecturas CTS $+I$ de la OEI, http://www.oei.es/salactsi/acevedo13.htm. Versión en castellano del capítulo 1 del libro de Manassero, M. A., Vázquez, A. y Acevedo, J. A.: Avaluació dels temes de ciència, tecnologia i societat. Palma de Mallorca: Conselleria d'Educació i Cultura del Govern de les Illes Ballears, 2001.

ACEVEdo, J. A., VÁzQUEZ, A. y MANASSERO, M. A. (2003). Papel de la educación CTS en una alfabetización científica y tecnológica para todas las personas. Revista Electrónica de Enseñanza de las Ciencias, 2(2), http://www.saum.uvigo.es/reec/.

ACEVEdo, J. A., VÁzQUeZ, A., MANASSERO, M. A. y ACEVEdo, P. (2003). Creencias sobre la tecnología y sus relaciones con la ciencia. Revista Electrónica de Enseñanza de las Ciencias, 2(3), http://www.saum.uvigo.es/reec/. 
ACEVEdo, J. A., VÁzQUez, A., MANASSERo, M. A. y ACEVEdo, P. (2007). Consensos sobre la naturaleza de la ciencia: fundamentos de una investigación empírica. Revista Eureka sobre Enseñanza y Divulgación de las Ciencias, 4(1), 42-66, http://www.apac-eureka.org/revista/Larevista.htm.

ACEVEDO, J. A. VÁZQUEZ, A., MARTÍN-GORDILlO, M., OLIVA, J. M., ACEVEDO, P., PAIXÃO, F., MANASSERO, M. A. (2005). La naturaleza de la ciencia y la educación científica para la participación ciudadana. Una revisión crítica. Revista Eureka sobre Enseñanza y divulgación de las Ciencias, 2(2), 121-140, http://www.apac-eureka.org/revista/Larevista.htm.

AIKENHEAD, G. S. (2003a). Review of Research on Humanistic Perspectives in Science Curricula. Paper presented at the $4^{\text {th }}$ Conference of the European Science Education Research Association (ESERA), Research and the Quality of Science Education. Noordwijkerhout, The Netherlands (august 19-23). En http://www.usask.ca/education/people/aikenhead/ESERA 2.pdf.

AIKENHEAD, G. S. (2003b). STS Education: A Rose by Any Other Name. En R. Cross (Ed.), A Vision for Science Education: Responding to the work of Peter J. Fensham (pp. 59-75). New York: Routledge Falmer. En http://www.usask.ca/education/people/aikenhead/stsed.pdf.

AIKENHEAD, G. S. (2005a). Educación Ciencia-Tecnología-Sociedad (CTS): una buena idea como quiera que se le llame. Educación Química, 6(2), 114-124.

AIKENHEAD, G. S. (2005b). Research into STS Science Education. Educación Química, 6(3), 384-397, 2005.

BANET, E. (2007). Finalidades de la educación científica en secundaria: opinión del profesorado sobre la situación actual. Enseñanza de las Ciencias, 25(1), 5-20.

BARTHOLOMEW, H., OSBORNE, J. y RATCLIFFE, M. (2004). Teaching Students "IdeasAbout-Science": Five Dimensions of Effective Practice. Science Education, 88(5), 655-682.

BELL, R. L. (2005). The Nature of Science in Instruction and Learning. Paper presented at the Annual International Conference of the Association for the Education of Teachers in Science (AETS), Colorado Springs, CO.

BYBEE, R. W. (1997). Achieving scientific literacy: From purposes to practices. Portsmouth, NH: Heinemann.

CAJAS, F. (2001). The Science/Technology Interaction: Implications for Science Literacy. Journal of Research in Science Teaching, 38(7), 715-729.

DOMÈNECH, J. L., GIL-PÉREZ, D., GRAS, A. y VILCHES, A. (2006). Per què una Dècada de I'Educació per un Futur Sostenible (2005-2014)? Crida de Nacions Unides a tots els Educadors. La Rella, 19, 265-288.

DRIVER, R., LEACH, J., MILLAR, R. y SCOTT, P. (1996). Young people's images of science. Buckingham: Open University Press.

FENSHAM, P. J. (2002a). De nouveaux guides pour l'alphabetisation scientifique. Canadian Journal of Science, Mathematics, and Technology Education, 2(2), 133-150.

FENSHAM, P. J. (2002b). Time to Change Drivers for Scientific Literacy. Canadian Journal of Science, Mathematics, and Technology Education, 2(1), 9-24.

FENSHAM, P. J. (2004). Beyond Knowledge: Other Scientific Qualities as Outcomes for School Science Education. En R. M. Janiuk y E. Samonek-Miciuk (Ed.), Science 
and Technology Education for a Diverse World - dilemmas, needs and partnerships (pp. 23-25). International Organization for Science and Technology Education (IOSTE) $\mathrm{XI}^{\text {th }}$ Symposium Proceedings. Lublin, Polland: Maria Curie-Sklodowska University Press.

FENSHAM, P. J. (2005). Interest in Science: Lessons and non-lessons from TIMSS and PISA. Paper presented at the $5^{\text {th }}$ Conference of the European Science Education Research Association (ESERA), Contributions of Research to Enhancing Students' Interest in Learning Science. Barcelona, Spain (26 august - 1 september).

FOUREZ, G. (1999), L'enseignement des sciences: en crise? La Revue Nouvelle, 110 (juillet-août), 96-99.

FOUREZ, G. (2002). Les sciences dans l'enseignement secondaire. Didaskalia, 21, 107-122.

FURIÓ, C., VILCHES, A., GUISASOLA, J. y ROMO, V. (2001). Finalidades de la enseñanza de las ciencias en la Secundaria Obligatoria. ¿Alfabetización científica o preparación propedéutica? Enseñanza de las Ciencias, 19(3), 365376.

GAGO, J. M. (Coord.) (2004). Increasing human resources for science and technology in Europe. Report presented at the European Community conference Europe Needs More Scientists. Bruselas, UE (2 de abril de 2004), http://europa.eu.int/comm/research/conferences/2004/sciprof/publications en. html.

GARDNER, P. L. (1975). Attitudes to science: A review. Studies in Science Education, $2,1-41$.

GARDNER, P. L. (1990). The technology-science relationship: Some curriculum implications. Research in Science Education, 20, 124-133.

GARDNER, P. L. (1994). Representations of the relationship between science and technology in the curriculum. Studies in Science Education, 24, 1-28.

GARDNER, P. L. (1999). The representation of science-technology relationships in Canadian physics textbooks. International Journal of Science Education, 21(3), 329-347.

GAULD, C. F. y HUKINS, A. A. (1980). Scientific attitudes: A review. Studies in Science Education, 7, 129-161.

GILBERT, J. K. (1992). The interface between science education and technology education. International Journal of Science Education, 14(5), 563-578.

GIL-PÉREZ, D. y VILCHES, A. (2006a). Algunos obstáculos e incomprensiones en torno a la sostenibilidad. Revista Eureka sobre Enseñanza y Divulgación de las Ciencias, 3(3), 507-516, http://www.apac-eureka.org/revista/Larevista.htm.

GIL-PÉREZ, D. y VILCHES, A. (2006b). ¿Cómo puede contribuir el proyecto PISA a la mejora de la enseñanza de las ciencias (y de otras áreas de conocimiento)? Revista de Educación, no extra, marzo, 295-311.

GIL-PÉREZ, D., VILCHES, A. y OLIVA, J. Ma (2005). Década de la educación para el desarrollo sostenible. Algunas ideas para elaborar una estrategia global. Revista Eureka sobre Enseñanza y Divulgación de las ciencias, 2(1), 91-100. http://www.apac-eureka.org/revista/Larevista.htm. 
GIL-PÉREZ, D., VILCHES, A., TOSCANO, J. C. y MACÍAS, O. (2006). Década de la educación para un futuro sostenible (2005-2014): Un punto de inflexión necesario en la atención a la situación del planeta. Revista Iberoamericana de Educación, 40, 125-178.

GUTIÉRREZ, A. (2006). PISA y la evaluación de la alfabetización científica. Investigación en la Escuela, 60, 65-78.

HALADYNA, T. y SHAUGHNESSY, J. (1982). Attitudes toward science: A quantitative synthesis. Science Education, 66(4), 547-563.

INECSE-MEC (2005a). Preguntas planteadas en PISA 2000. Lectura, matemáticas y ciencias. Madrid: INECSE/MEC, http://www.ince.mec.es/index.htm.

INECSE-MEC (2005b). Programa PISA. Ejemplos de ítems de Conocimiento Científico. Madrid: INECSE/MEC, http://www.ince.mec.es/index.htm.

JENKINS, E. W. (2006a). Student opinion in England about science and technology. Research in Science \& Technological Education, 24(1), 59-68, http://www.ils.uio.no/english/rose/network/countries/uk-england/eng-jenkinsrste2006.pdf.

JENKINS, E. W. (2006b). The Student Voice and School Science Education. Studies in Science Education, 42, 49-88, http://www.ils.uio.no/english/rose/network/countries/uk-england/eng-jenkinssse2006.pdf.

JENKINS, E. W. y NELSON, N. W. (2005). Important but not for me: students' attitudes towards secondary school science in England. Research in Science \& Technological Education, 23(1), 41-58.

KLOPFER, L. E. (1971) Evaluation of Learning in Science. En B. Bloom, J. Hastings y G. Manaus (Eds.), Handbook of Summative and Formative Evaluation of Student Learning. New York: McGraw-Hill.

KLOPFER, L. E. (1976). A structure for the affective domain in relation to science education. Science Education, 60(3), 299-312.

LAFORGIA, J. (1988). The affective domain related to science and its evaluations. Science Education, 72(4), 407-421.

LAVONEN, J., BYMAN, R., JUUTI, K., MEISALO, V. y UITTO, A. (2005). Pupil Interest in Physics: A Survey in Finland. Nordina, 2, 72-85, http://www.ils.uio.no/english/rose/network/countries/finland/fin-lavonennordina2005.pdf.

LEDERMAN, N. G. (2006). Research on Nature of Science: Reflections on the Past, Anticipations of the Future. Asia-Pacific Forum on Science Learning and Teaching, 7(1), http://www.ied.edu.hk/apfslt/v7 issue1/foreword/index.htm.

MAIZTEGUI, A., ACEVEDO, J. A., CAAMAÑO, A., CACHAPUZ, A., CAÑAL, P., CARVALHO, A. M. P., DEL CARMEN, L., DUMAS CARRÉ, A., GARRITZ, A., GIL, D., GONZÁLEZ, E., GRAS-MARTÍ, A., GUISASOLA, J., LÓPEZ-CEREZO, J. A., MACEDO, B., MARTÍNEZ-TORREGROSA, J., MORENO, A., PRAIA, J., RUEDA, C., TRICÁRICO, H., VALDÉS, P. y VILCHES, A. (2002). Papel de la tecnología en la educación científica: una dimensión olvidada. Revista Iberoamericana de Educación, 28, 129-155, http://www.rieoei.org/rie28a05.PDF.

McCOMAS, W. F. (Ed.) (1998). The nature of science in science education: Rationales and strategies. Dordrecht, The Netherlands: Kluwer Academic Publishers. 
MILLAR, R. y OSBORNE, J. (Eds.) (1998). Beyond 2000. Science education for the future. London: King's College London School of Education.

OECD (2000). Measuring student knowledge and skills: The PISA assessment of reading, mathematical and scientific literacy. París: OECD, http://www.pisa.oecd.org/. Traducción de G. Gil Escudero, J. Fernández García, F. Rubio Miguelsanz, C. López Ramos y S. Sánchez Robles (2001), La medida de los conocimientos y las destrezas de los alumnos: La evaluación de la lectura, las matemáticas y las ciencias en el proyecto PISA 2000. Madrid: INCE/ MECD, http://www.ince.mec.es/index.htm.

OECD (2003a). The PISA 2003 Assessment Framework: Mathematics, Reading, Science and Problem Solving Knowledge and Skills. París: OECD, http://www.pisa.oecd.org/. Traducción de E. Belmonte (2004), Marcos teóricos de PISA 2003: la medida de los conocimientos y destrezas en matemáticas, lectura, ciencias y resolución de problemas. Madrid: INECSE/MEC, http://www.ince.mec.es/index.htm.

OECD (2003b). Definition and Selection of Competencies: Theoretical and Conceptual Foundations (DeSeCo). Summary of the final report "Key Competencies for a Successful Life and a Well-Functioning Society". París: OECD.

OECD (2006). Assessing Scientific, Reading and Mathematical Literacy: A Framework for PISA 2006. París. OECD, http://www.pisa.oecd.org/. Traducción castellana (2006), Evaluación de la Competencia Científica, Lectora y Matemática: Un marco teórico para PISA 2006. Madrid: INECSE/MEC, http://www.ince.mec.es/index.htm.

OLIVA, J. M. (2006). Editorial. Revista Eureka sobre Enseñanza y Divulgación de las Ciencias, 3(1), 1, http://www.apac-eureka.org/revista/Larevista.htm.

OLIVA, J. M. (2007). Editorial. Revista Eureka sobre Enseñanza y Divulgación de las Ciencias, 4(1), 1, http://www.apac-eureka.org/revista/Larevista.htm.

OLIVA, J. M. y ACEVEDO, J. A. (2005). La enseñanza de las ciencias en primaria y secundaria hoy. Algunas propuestas de futuro. Revista Eureka sobre Enseñanza y Divulgación de las Ciencias, 2(2), 142-151, http://www.apaceureka.org/revista/Larevista.htm.

OSBORNE, J., COLLINS, S., RATCLIFFE, M., MILLAR, R. y DUSCHL, R. (2003). What "Ideas-about-Science" Should Be Taught in School Science? A Delphi Study of the Expert Community. Journal of Research in Science Teaching, 40(7), 692720.

OSBORNE, J., SIMON, S. y COLLINS, S. (2003). Attitudes towards science: a review of the literature and its implications. International Journal of Science Education, 25(9), 1049-1079.

PAJARES, R. (2005). Resultados en España del estudio PISA 2000: conocimientos y destrezas de los alumnos de 15 años. Madrid: INECSE/MEC, http://www.ince.mec.es/index.htm.

REISS, M. J. (2006). Desarrollo de un curso de biología contextualizado en el bachillerato: el caso del proyecto Salters-Nuffield Advanced Biology. Enseñanza de las Ciencias, 24(3), 429-438.

SCHIBECI, R. A. (1984). Attitudes to science: An update. Studies in Science Education, 11, 26-59. 
SCHREINER, C. (2006). Exploring a ROSE-garden: Norwegian youth's orientations towards science - seen as signs of late modern identities. Doctoral thesis. Department of Teacher Education and School Development, Faculty of Education, University of Oslo, http://www.ils.uio.no/english/rose/network/countries/norway/eng/norschreiner-thesis.pdf.

SCHREINER, C. y SJøBERG, S. (2004a). Do pupils think they can change the world? Comparative data on environmental challenges seen in the light of recent perspectives from youth research. En R. M. Janiuk y E. Samonek-Miciuk (Eds.), Science and Technology Education for a Diverse World - dilemmas, needs and partnerships (pp. 209-210). International Organization for Science and Technology Education (IOSTE). XI ${ }^{\text {th }}$ Symposium Proceeding. Lublin, Poland: Marie Curie-Sklodowska University Press.

SCHREINER, C. y SJØBERG, S. (2004b). ROSE: The relevance of science education. Sowing the seeds of ROSE. Background, Rationale, Questionnaire Development and Data Collection for ROSE (The Relevance of Science Education) - a comparative study of students' views of science and science education. Acta didactica 4, University of Oslo, Norway, Faculty of Education, Department of Teacher Education and School Development, http://www.ils.uio.no/english/rose/key-documents/key-docs/ad0404-sowingrose.pdf.

SCHREINER, C. y SJØBERG, S. (2005). Empowered for action? How do young people relate to environmental challenges? En S. Alsop (2005), The affective dimensions of cognition: Studies from education in the Sciences (cap. 11). Dordrecht, The Netherlands: Kluwer Academic Publishers, http://www.ils.uio.no/english/rose/network/countries/norway/eng/norschreiner-springer2004.pdf.

SJØBERG, S. (2004). Science Education: The voice of the learners. Contribution to the Conference on Increasing Human Resources for Science and Technology in Europe. Bruselas, UE (2 de abril de 2004), http://europa.eu.int/comm/research/conferences/2004/sciprof/pdf/sjoberg.pdf y $\quad$ http://www.ils.uio.no/english/rose/network/countries/norway/eng/norsjoberg-eu2004.pdf.

SJØBERG, S. y SCHREINER, C. (2005a). How do learners in different cultures relate to science and technology? Results and perspectives from the project ROSE (The Relevance of Science Education). Asia-Pacific Forum on Science Learning and Teaching, 6(2), http://www.ied.edu.hk/apfslt/v6 issue2/foreword/index.htm.

SJøBERG, S. y SCHREINER, C. (2005b). Perceptions and images of science and science education Some results from the ROSE project, a comparative project in 32 countries. Paper presented at the $5^{\text {th }}$ Conference of the European Science Education Research Association (ESERA), Contributions of Research to Enhancing Students' Interest in Learning Science. Barcelona, Spain (26 August - 1 September), CD-ROM Edition.

SJøBERG, S. y SCHREINER, C. (2005c). Young people and science. Attitudes, values and priorities. Evidence from the ROSE project. Keynote presentation at EU's Science and Society Forum 2005. Session 4: How to foster diversity, 
inclusiveness and equality in science. Bruselas, UE (9-11 de abril de 2005), http://europa.eu.int/comm/research/conferences/2005/forum2005/docs/progr sjoberg en.pdf

http://www.ils.uio.no/english/rose/network/countries/norway/eng/nor-sjobergeu2005.pdf.

SOLANO-FLORES, G., CONTRERAS-NIÑO, L. A. y BACKHOFF-ESCUDERO, E. (2006). Traducción y adaptación de pruebas: Lecciones aprendidas y recomendaciones para países participantes en TIMSS, PISA y otras comparaciones internacionales. Revista Electrónica de Investigación Educativa, 8(2), http://redie.uabc.mx/vol8no2/contenido-solano2.html.

SOLANO-FLORES, G. y NELSON-BARBER, S. (2001). On the cultural validity of science assessments. Journal of Research in Science Teaching, 38(5), 553-573.

TRUMPER, R. (2006a). Factors Affecting Junior High School Students' Interest in Biology. Science Education International, 17(1), 31-48, http://www.ils.uio.no/english/rose/network/countries/israel/isr-trumpersei2006.pdf.

TRUMPER, R. (2006b). Factors Affecting Junior High School Students' Interest in Physics. Journal of Science Education and Technology, 15(1), 47-58, http://www.ils.uio.no/english/rose/network/countries/israel/isr-trumperphysintr2006.pdf.

UNESCO (2003). UNESCO and the International Decade of Education for Sustainable Development (2005-2015). UNESCO International Science, Technology and Environmental Education Newsletter, XXVIII (1-2). París: UNESCO.

UNESCO (2005). International Implementation Scheme for the UN Decade of Education for Sustainable Development. París: UNESCO.

VALDÉS, P., VALDÉS, R., GUISASOLA, J. y SANTOS, T. (2002). Implicaciones de las relaciones ciencia-tecnología en la educación científica. Revista Iberoamericana de Educación, 28, 101-128.

VÁzQUEZ, A., ACEVEDO, J. A. y MANASSERO, M. A. (2005). Más allá de una enseñanza de las ciencias para científicos: hacia una educación científica humanística. Revista Electrónica de Enseñanza de las Ciencias, 4(2), http://www.saum.uvigo.es/reec/.

VÁzQUEZ, A., ACEVEdo, J. A., MANASSERo, M. A. y ACEVEdo, P. (2001). Cuatro paradigmas básicos sobre la naturaleza de la ciencia. Argumentos de Razón Técnica, 4, 135-176. Consultado 4/1/2007 en Sala de Lecturas CTS+I de la OEI, 2003, http://www.oei.es/salactsi/acevedo20.htm.

VÁZQUEZ, A. y MANASSERO, M. A. (1995). Actitudes relacionadas con la ciencia: una revisión conceptual. Enseñanza de las ciencias, 13(3), 337-346.

VÁZQUEZ, A. y MANASSERO, M. A. (2004). Imagen de la ciencia y la tecnología al final de la educación obligatoria. Cultura y Educación, 16(4), 385-398, http://www.ils.uio.no/english/rose/network/countries/spain/esp-vazquez2004.pdf.

VÁZQUEZ, A. y MANASSERO, M. A. (2005). Actitudes de los jóvenes en relación con los desafíos medio-ambientales. Infancia y Aprendizaje, 28(3), 309-327, http://www.ils.uio.no/english/rose/network/countries/spain/esp-vazquez2005.pdf. 
VÁZQUEZ, A. y MANASSERO, M. A. (2006). El interés de los estudiantes hacia la Química. Educación Química, 17(3), 388-401.

VÁZQUEZ, A. y MANASSERO, M. A. (2007). En defensa de las actitudes y emociones en la educación científica (I): evidencias y argumentos generales. Revista Eureka sobre Enseñanza y Divulgación de las Ciencias, 4(2), 247-271, http://www.apac-eureka.org/revista/Larevista.htm.

VILCHES, A. y GIL-PÉREZ, D. (2003). Construyamos un futuro sostenible. Diálogos de supervivencia. Madrid: Cambridge University Press. 


\section{THE SCIENCE AND TECHNOLOGY ATTITUDES RELATED IN THE PISA 2006 STUDY}

\section{SUMMARY}

This paper describes the main changes incorporated in the international comparative evaluation PISA 2006. After analyze the adopted meaning of scientific literacy, we paid a special attention to the evaluation of the science and technology attitudes related, in order of point out the aspects that it evaluates this new dimension, and to how the PISA 2006 Project makes it.

Key words: External evaluation, PISA 2006 project, scientific literacy, science \& technology related attitudes. 